\section{Giving your SEM or FIB a Helping Hand}

Neil Rowlands, Oxford Instruments, Concord, USA.

Gavin Frayne, Kleindiek Nanotechnik, Tubingen, Germany.

Bo Svarrer Hansen, Capres A/S, Lyngby, Denmark. neil.rowlands@oxinst.com

\section{INTRODUCTION}

Scanning electron microscopes have traditionally been used for observation and microanalysis of samples. Positioning and testing of samples has usually been performed out of the SEM chamber e.g. electrical test benches, sample preparation. However, due to miniaturization in semiconductor technology, optics, micro-mechanics, medicine, gene- and biotechnology, highly precise positioning techniques are becoming increasingly important. This may be performed using an optical microscope, or more commonly, within the SEM chamber itself.

Positioning may be accomplished either by moving the sample or moving the stage. The need for very accurate positioning when using e-beam writing techniques and precision alignment of extremely fine probes for failure analysis testing of $100 \mathrm{~nm}$ circuit technology has necessitated the need for ever smaller tools with nanometer accuracy for this work. In addition to probing and e-beam writing, it is often desirable to be able to move nano-particles such as carbon nanotubules (CNTs) or remove thin sections from FIB cuts and remount them on TEM grids. Furthermore, research into the nature of nano-particles often requires manipulation and testing on a very small scale.

\section{NANOMANIPULATORS}

The ability to conduct experiments in the SEM is becoming much more commonplace. The ability to move particles, test hardness, perform electrical tests and bring extremely small quantities of liquids and gases in contact with materials is essential for the evaluation of many new materials.

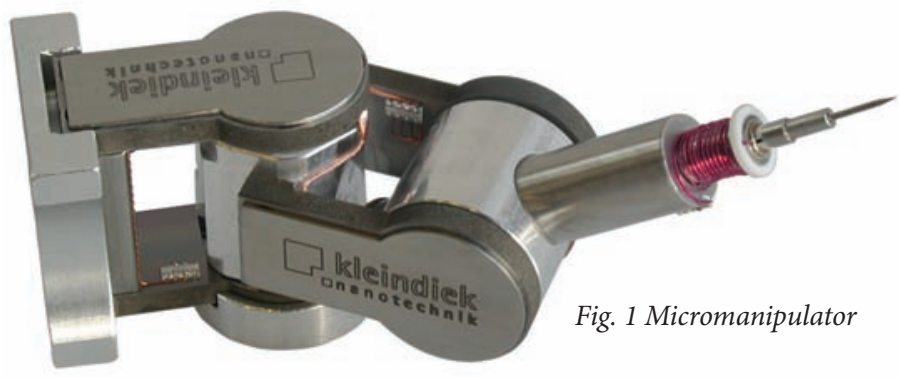

Manipulators that have the ability to perform a number of different tasks by simply changing the tip are valuable assets in many laboratory situations. Possibly the most common in-situ manipulation technique in common usage is the lift-out technique for TEM specimen preparation in Focused Ion Beam (FIB) tools. This entails FIB milling of samples down to very thin lamellae, which may then be lifted out of the milled trench and mounted on grids for TEM observation at high magnification. A number of techniques have been developed to do this but Figure 2 shows a method by which microgrippers are used to detach the lamella from the trough and then attach it to the side of a slot grid for evaluation in the TEM. This lessens the likelihood of losing the lamella during ex-situ transfer, as all operations are performed over a very small area in the FIB.

Manipulators may also be used for relocating or transferring samples in the FIB or SEM. Due to the fact that the manipulators are commonly not attached to the microscope stage, it is possible to lift samples with the aid of two manipulators and by moving the stage they can be relocated with ease.
This is particularly useful in the construction of nano engineered materials or NEMs devices where very small objects can be moved and deposited in a defined construction site. It may also be necessary to turn the particles over to view the other side. This may be accomplished using just one manipulator or a combination to turn the object without causing any damage. Manipulators have also been used to cleave and cut objects and also to separate agglomerates of particulates for further investigation, moving individual particles as necessary.

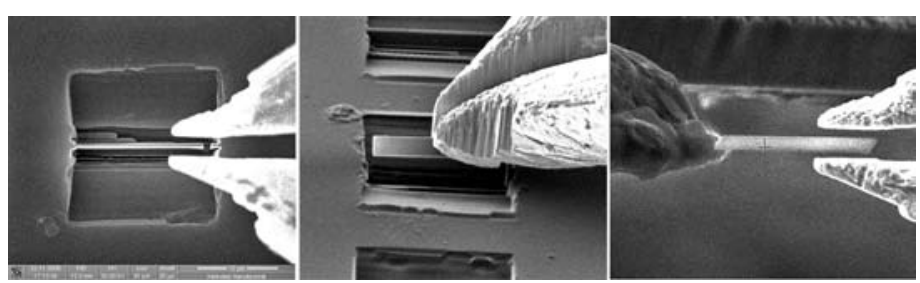

Fig 2a. Microgripper on lamella

Fig 2b. Removing the lamella

Fig 2c. Re-depositing lamella on a grid

Quite commonly tensile strength measurements can be performed by bonding one end of a CNT to a manipulator and measuring the force that is required to detach the tubule from the substrate. Likewise, the elastic modulus of tubules may be measured by using force measurement tips that can be added to the manipulator. These take the form of a cantilevered tip, somewhat similar to an AFM that can be used to measure force or nano-indentation. Tensile strength measurements have been made on many different materials including CNTs, beetle setae, spider silk and wood cell wall material. The force measurement tip can also be used for nano-indentation on a variety of materials including polymers, coatings, inclusions in steels, and aluminum samples.

Electrical properties of circuits may also be measured using a combination of two or more manipulators. A simple task in the world of electronics is the measurement of an I-V curve of a single transistor.

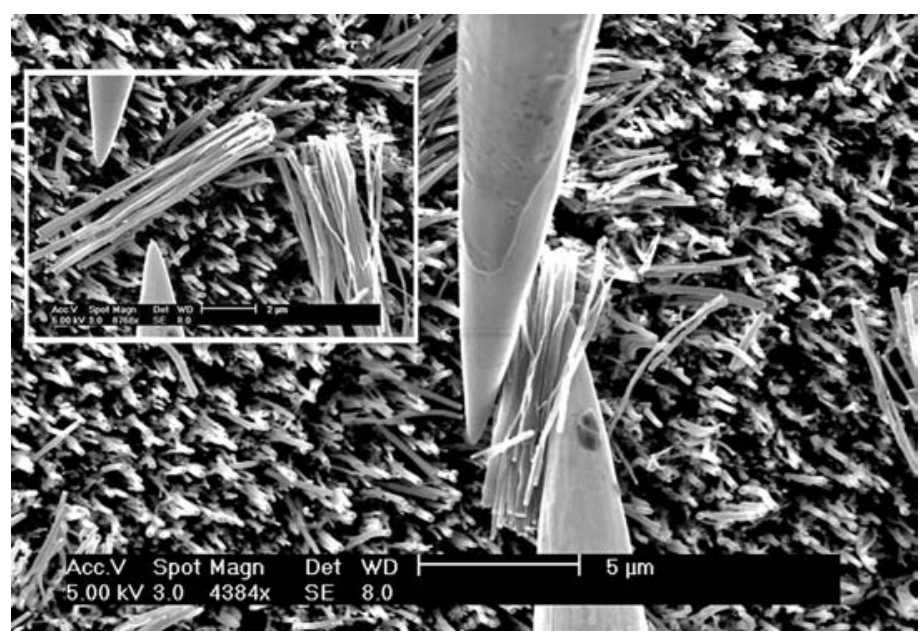

Fig 3. Picking up a bundle of CNTs with two manipulators

However, if the size of the transistor is reduced to a few hundred nanometers or less, this task becomes very challenging. Probing on small structures requires high positional accuracy and a very stable probing system. It is recommended that the probes have a positioning accuracy better than $5 \mathrm{~nm}$. and should drift no more that $1 \mathrm{~nm}$. per minute. These manipulators are commonly used in failure analysis of memory cells, transistors and other nanocircuitry. It is even 



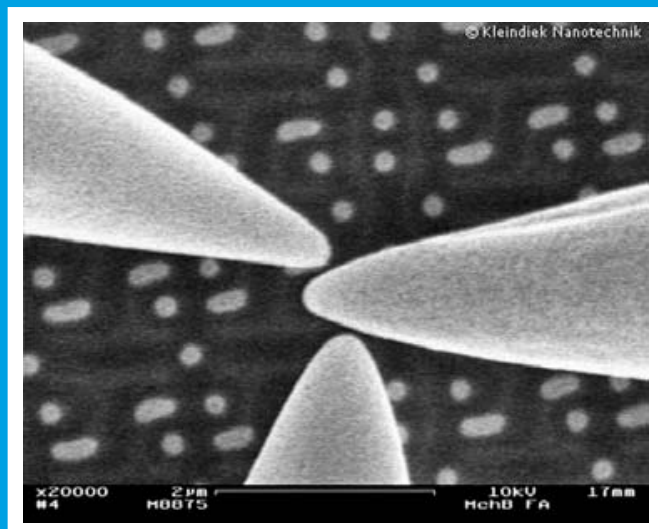

Fig 4. Probe tips on $100 \mathrm{~nm}$ contact pads of a transistor

within the sample and this current is then amplified for imaging purposes.

EBIC uses the current that arises from the generation of electron hole pairs within a sample while RCI only detects only those electrons remaining or diffusing to metal layers that are usually on top of the device. In order to conduct an EBIC or RCI investigation it is necessary to have one or two manipulators with highly conducting probe tips and an amplifying low-noise current-to-voltage converter that outputs directly to the video input of the microscope EBIC images are created from electrons with energies of more than $5 \mathrm{keV}$ that penetrate through the semiconductor's oxide and metal layers to the $p$ - $n$ junction. The electron hole pairs that are generated in this process are separated by the diffusion voltage and measured as a current that flows through the probe tip of the manipulator. The current signal is converted to a voltage and fed back to the SEM to obtain a current image of the sample. The light and dark areas in the image indicate $p$ - $n$ junctions ( $n$ - and $p$-wells to the substrate).

In the grey regions no current is flowing. A great advantage of EBIC is the ability to generate electron hole pairs at specific depths by selecting appropriate electron beam energies. The higher the beam energy, the deeper the penetration into the sample. By taking

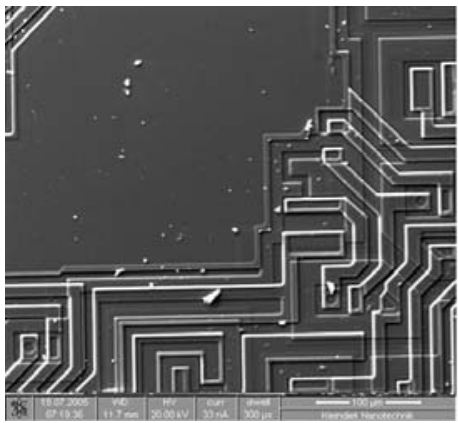

Fig 5a. SEM Image

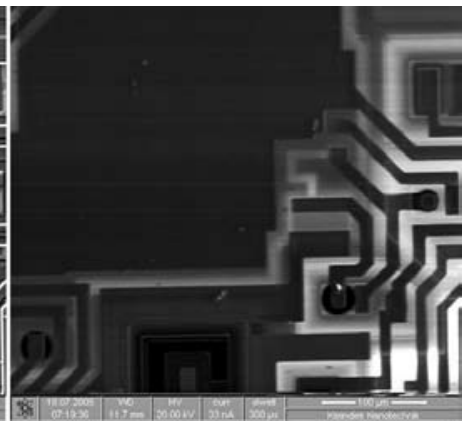

Fig 5b. EBIC Image pictures using different energies, a three-dimensional image of the $p-n$ junctions can be created. RCI differs from EBIC in that the current for the image is generated by electrons diffusing into metal wires that are being contacted by the probe needles of the manipulators. Typically, a contact is made with two wires that are spread over the whole area of interest. The result is a resistance image of the current flow from the electron beam position to the contact points. The higher the current, the brighter the image will be at the electron beam position. The beam energy is usually set below $5 \mathrm{kV}$ to avoid penetration down to the $p-n$ junctions. As the current is very small, a low-noise amplifier with high bandwidth is needed to get good images within a reasonable time.

In-situ four point probing is very useful in assessing the electrical properties of beam-induced depositions. These deposits are commonly laid down for circuit editing in FIBs and deposits may also be generated by exposing organo-metallic precursor gases to a focused electron beam (FEB). It is important to know the electrical properties such as resistance of these materials, especially in the case of metals that have been laid down to create local conductivity paths in modified circuits. The resistivity is measured by contacting the desired structure at four locations over its length. A forced current is then passed through the outer pins 1 and 4 and the voltage drop can be measured between pins 2 and 3 using a very high ohmic device so that the current between pin 2 and 3 is nearly zero. In this case the addition contact resistance does not play a role. An I/V curve may be generated to assess the behavior of the device, typically in the $\mu \mathrm{A}$ to $\mathrm{mA}$ range. If the resulting graph shows a straight line, the structure behaves as an ohmic resistor.
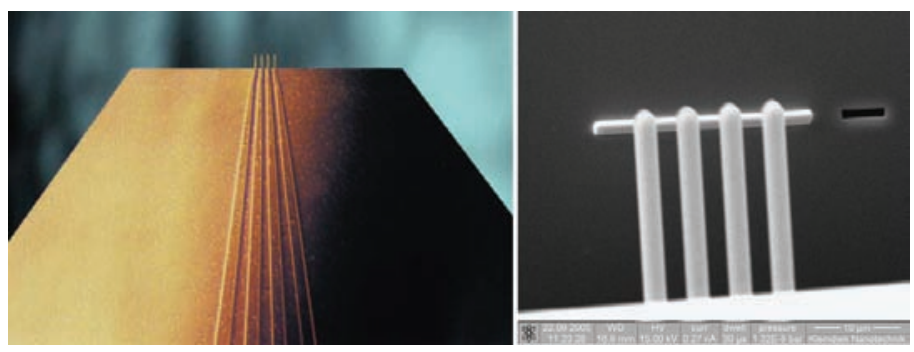

Fig 6. Four point probe viewed in an optical microscope

Fig 7. Four point probe on metal line viewed in the SEM

Another interesting application is direct measurements of resistance of carbon nanotubes where the combination of of the manipulator and the four point probe enables selection and electrical characterization of individual CNTs.

In general, microscopic four point probes are excellent for sheet resistance measurements and high resolution scans on conductive thin films.

Micro-injection can be used in environmental SEMs to demonstrate the effects of liquids and gases on materials on (a) a micro scale. Applications include the effect of liquids on the microstructure of textiles, dissolution effects on pharmaceutical products, assessing absorption properties of paper products and inks, liquid injection in foods and corrosion effects on cement, concrete and metals. Gases

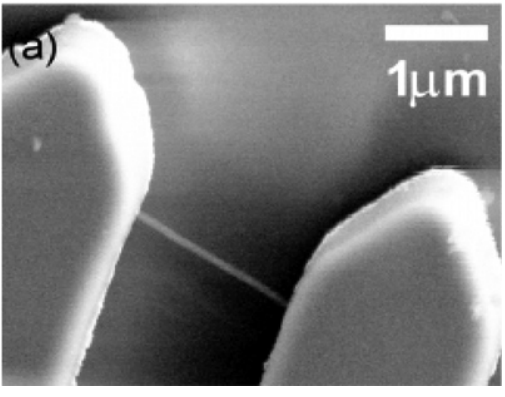

Fig 8. Four point probe on multi walled carbon nanotube may also be injected for electron beam deposition of materials in SEMs.

\section{STAGES}

High precision stages are necessary in situations where the original SEM stage does not have the required accuracy for the task in hand. Such uses include e-beam lithography, defect navigation and cell counting. Sub-stages are easily added to the original SEM and enhance the capability of the tool significantly. Added software provides automation for these sub stages and, with the aid of optical encoders, can record their position with nanometer accuracy. 


\section{An Automated Tissue Processor that canl do it ALL with ease.}

EMS is proud to announce the release of the most unique state-of-the-art tissue processor which not only is compatible with all plastic resins but paraffin waxes as well.
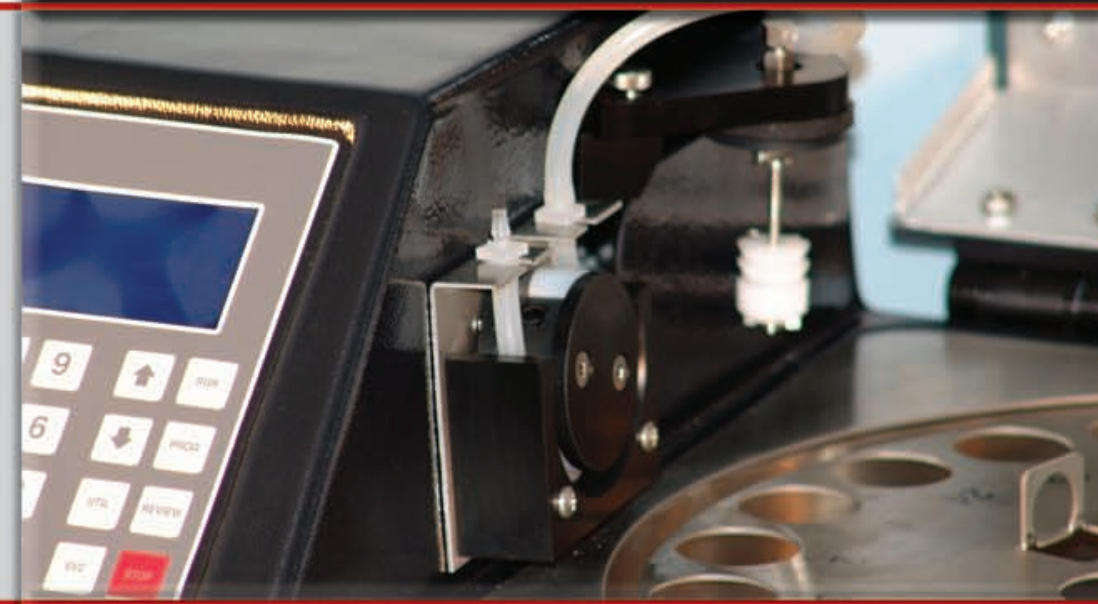

LYNX II is designed to be the successor of the Lynx Tissue Processor with several enhancements including capabilities to perform optional processing of larger size samples for Histology.
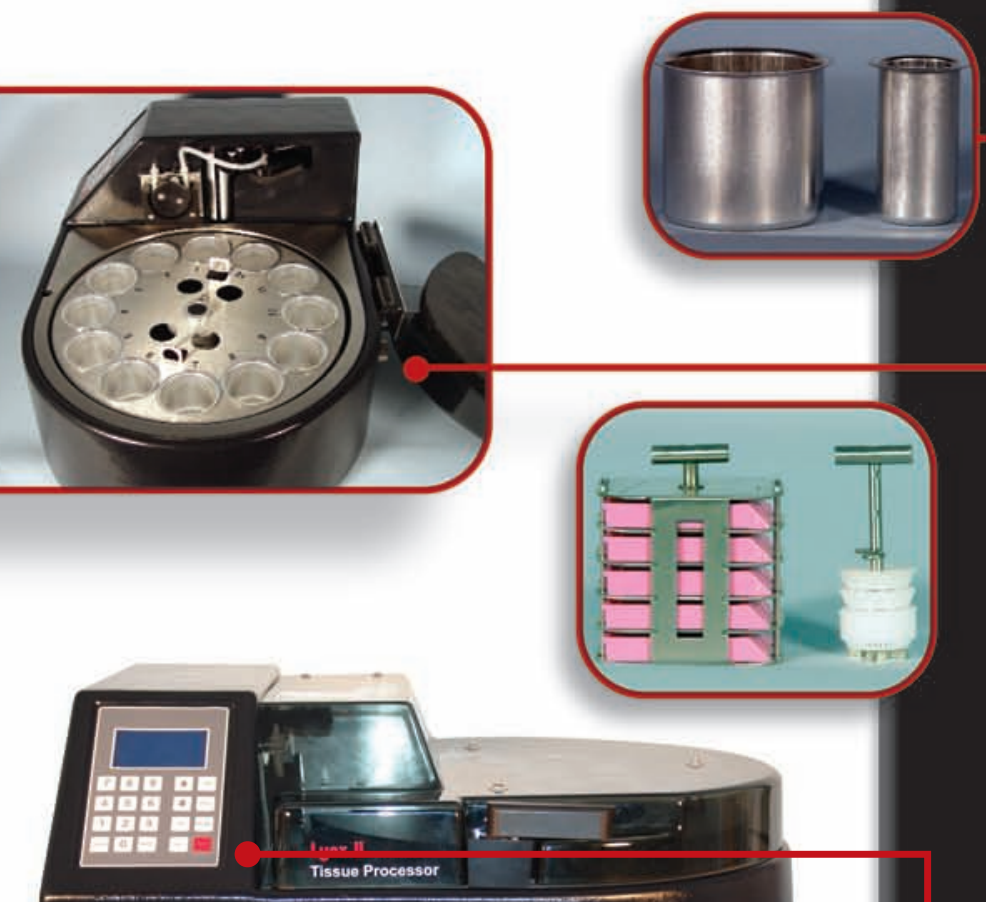

\section{Electron}

clescopy

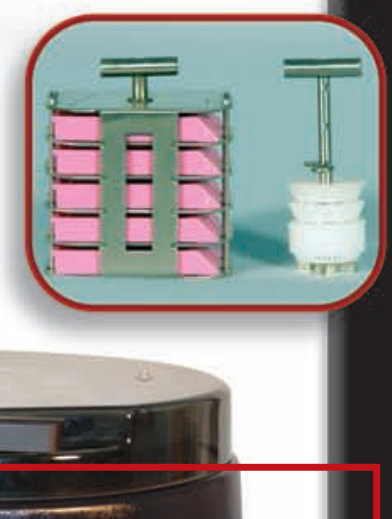

Electron

Microscopy Sciences $\square \quad \prod$ Automated Tissue Processor for Histology and Microscopy

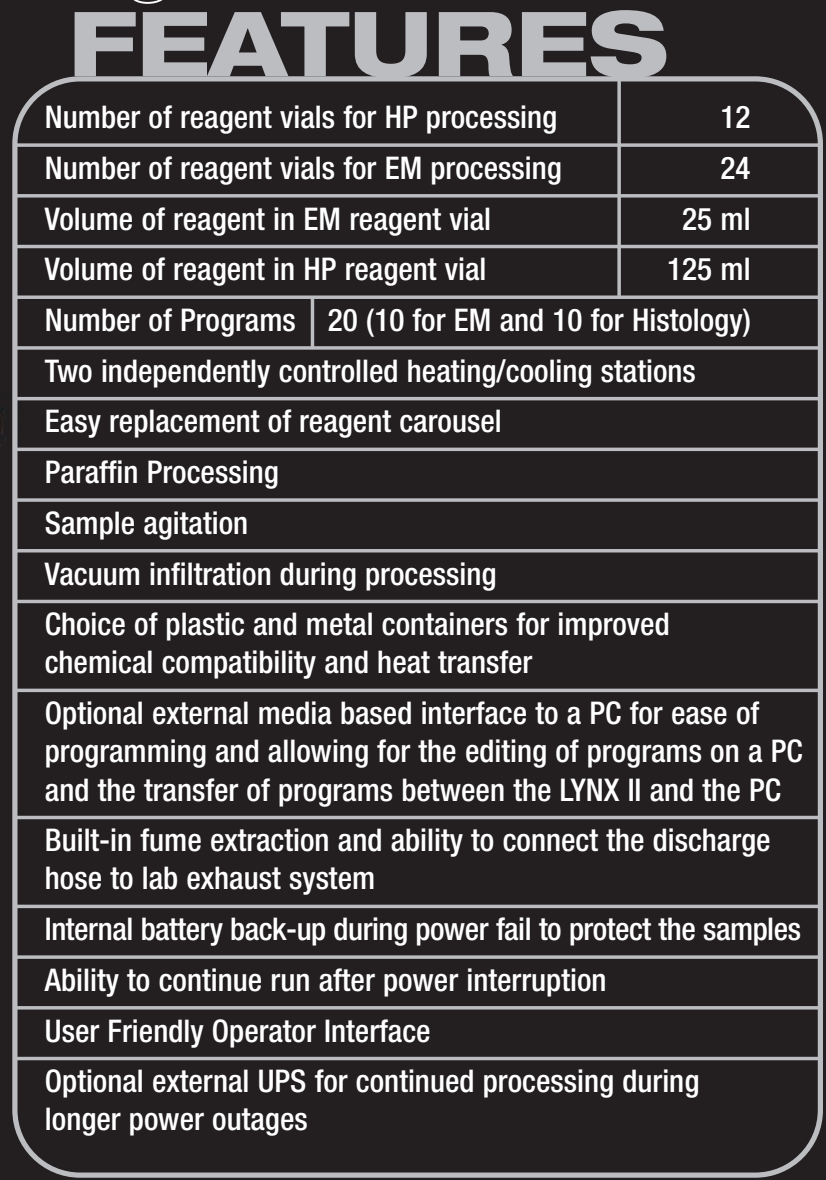

P.0. Box 550 - 1560 Industry Rd. • Hatfield, Pa 19440 (215) 412-8400 • Toll Free: 1-(800) 523-5874 Fax: (215) $412-8450$ or 8452

email: sgkcck@aol.com•stacie@ems-secure.com Website: www.emsdiasum.com www.electronmicroscopysciences.com 